\title{
An IGISOL portrait
}

\author{
J. Äystö • T. Eronen • A. Jokinen • A. Kankainen · \\ I. D. Moore · H. Penttilä
}

Published online: 16 May 2013

(C) Springer Science+Business Media Dordrecht 2013

This issue of Hyperfine Interactions consists of 21 articles describing scientific work related to the research at the on-line isotope separator facility IGISOL at the University of Jyväskylä, Finland. Ten contributions containing a significant amount of original results, earlier unpublished, have been presented as a Topical Collection in the European Physical Journal (EPJA 48 issue No 4, 2012).

In the early 1980s, the Ion Guide Isotope Separator On-Line (IGISOL) concept was realized at the $20 \mathrm{MeV}$ proton cyclotron of the Physics Department at the University of Jyväskylä (JYFL). For the first time, the technique allowed the production of high quality ISOL beams from nuclear reaction products with millisecond delay times without chemical restrictions. By providing access to short-lived isotopes of refractory elements it opened up new regions in the nuclear chart for nuclear decayand ground-state spectroscopy.

During the 1980s, the main research activity at IGISOL was decay spectroscopy, and in particular, of fission products produced in proton-induced fission of natural uranium. Approximately 40 new isotopes and isomers were discovered and studied employing beta-, gamma-, and conversion electron spectroscopy. These experiments covered a broad range of neutron-rich nuclei from yttrium $(Z=39)$ to cadmium $(Z=48)$ and revealed how their structure was developing between highly deformed zirconium through triaxial ruthenium isotopes to weakly deformed vibrational cadmium isotopes.

The original IGISOL facility was shut down in October 1991 and was reinstalled as IGISOL-2 at the science campus housing the laboratory for the $\mathrm{K}=130 \mathrm{MeV}$ heavy ion cyclotron. A new window of opportunity for extending research to neutrondeficient nuclei far from stability was opened and was going to have a strong impact

Laboratory portrait. Three decades of research using IGISOL technique at the University of Jyväskylä, Finland, 2010.

J. Äystö · T. Eronen · A. Jokinen · A. Kankainen · I. D. Moore · H Penttilä ( $ه)$

Department of Physics, University of Jyväskylä, P.O. Box 35, FI-40014 Jyväskylä, Finland

e-mail: heikki.penttila@jyu.fi 
in extending the scope of the IGISOL research program. The first two decades of IGISOL was reviewed in Nuclear Physics A 609 (2001) 477.

At the start of the new millennium novel ion trap installations were introduced at the IGISOL facility. The radiofrequency cooler and buncher trap (RFQ) was installed to improve the beam quality for the collinear laser spectroscopy station. Furthermore, the ability to bunch the ion beam dramatically suppressed the scattered photon background, a technique which has since been adapted at other international facilities. The drive to produce isobarically clean beams was realized with the construction of a longitudinal Penning trap of which the coupling to a precision trap would later lead to a very successful mass measurement programme.

In 2003 the front end of the separator was upgraded, implying the birth of the IGISOL-3 facility. With improved yields and the aid of the new instrumentation, precision experiments on both neutron-rich fission products as well as neutron-deficient nuclei produced in light- and heavy-ion fusion reactions could be performed. The experiments covered both decay spectroscopy, often enhanced by Penning trap purification of the reaction products, and accurate measurements of ground state properties such as charge radii and masses.

In particular, the Penning trap mass spectroscopy of the neutron-rich fission products and neutron-deficient fusion-evaporation residues resulted in improved atomic mass values for over 300 isotopes or isomeric states. These measurements revealed large inaccuracies in adopted atomic masses, thus having influence on the future development of theoretical mass models. Precisely measured two-neutron separation energies allowed the investigation of shape transitions around $N=60$ and evolution of shell gaps at $N=50$ and $N=82$. These measurements were often supported by both laser- and decay spectroscopy studies, thus providing a complementary picture of the evolution of nuclear structure of exotic nuclei.

Operation at IGISOL-3 was stopped in June 2010. The whole facility was moved and re-assembled in a new accelerator hall coupled to a new, high-intensity $30 \mathrm{MeV}$ proton cyclotron MCC30. Primary beams from both MCC30 and the K=130 heavyion cyclotron are available for delivery to the IGISOL target chamber. Since the MCC30 cyclotron is almost solely dedicated for IGISOL, time available for experiments is significantly increased. The main improvements of the new facility include considerably increased floor space for future developments and more sophisticated detector setups. Optical transportation to the target area for laser ionization has been significantly improved. The RFQ trap is now located in the central beam line, where it can be better accessed with laser beams for optical manipulation, ion resonance ionization or polarization of ion beams. The gas handling system has been totally renovated, and an independent off-line ion source beam line is currently under construction. The design of the radiation shielding and the beam transportation has been modified to fully benefit the available primary beam intensities for experiments at the new IGISOL facility, IGISOL-4. 


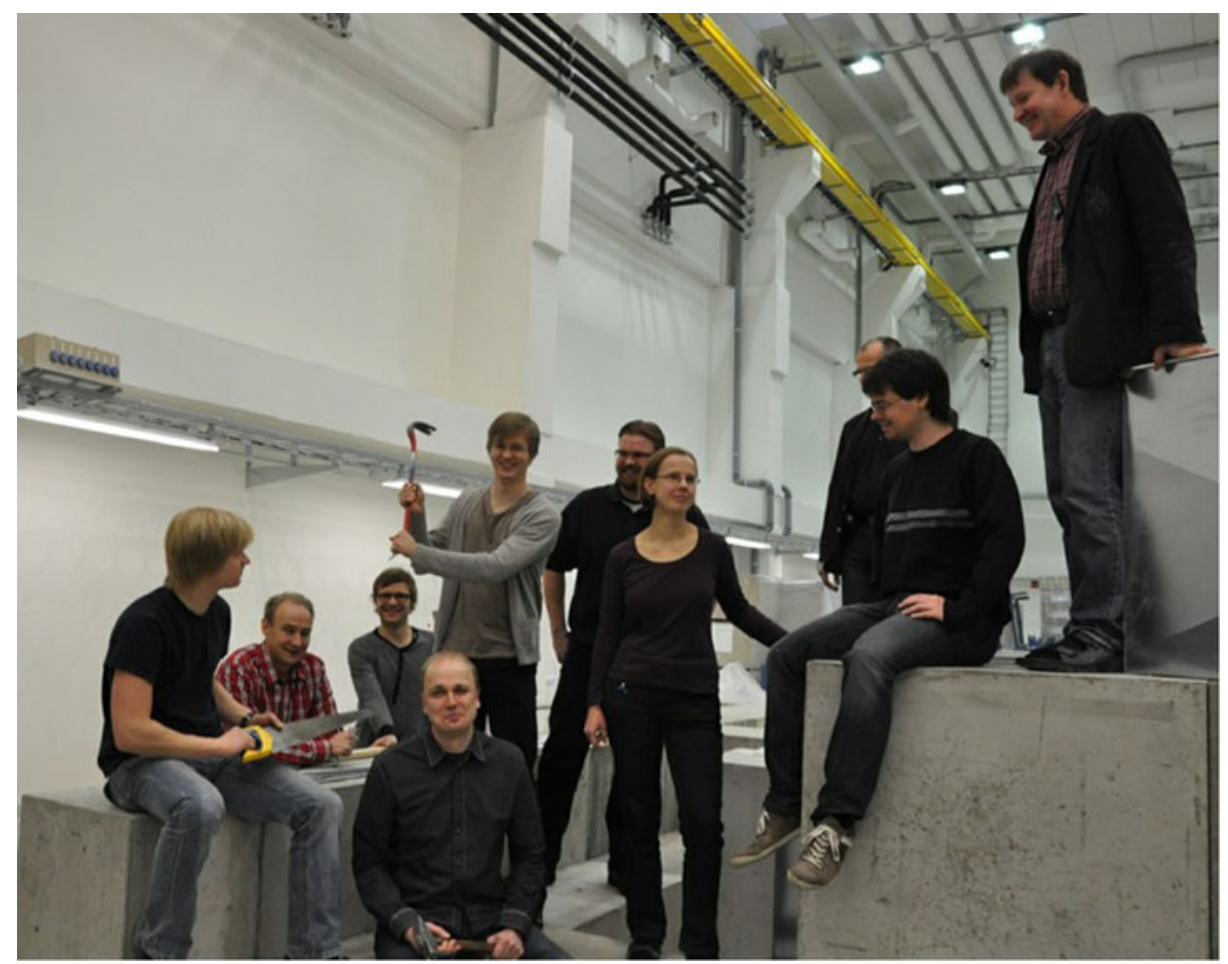

